\title{
ON ORDERING INFINITELY MANY SMALL HOMEOMORPHISMS
}

\author{
JOHN COBB
}

A familiar technique in topology is that of performing a finite sequence of "adjustments" of some sort, each the identity except in a small set. In case infinitely many adjustments are needed-e.g., when the adjusting is done modulo a closed subset-convergence problems arise: a point may be moved infinitely often and not approach a limiting position; or a converging sequence might be torn away from its limit point. This paper provides a method of ordering the domains of the adjustments such that convergence is automatic if the adjustments are carried out in the prescribed order.

All orderings are by sequences of positive integers. A chain is a sequence of sets in which adjacent elements intersect; others may also interesect. The length of a chain is the number of elements in it. In a metric space, the diameter of a chain is the diameter of the union of all sets in the chain. If $U$ is an ordered collection of sets, a chain of elements of $U$ is said to be ascending (with respect to the ordering on $U$ ) if the chain ordering coincides with the $U$-ordering on the elements of the chain. The ordering on $U$ satisfies the ascending chain condition (a.c.c.) if each ascending chain is finite.

Consider the partition of $E^{n}$ by the complete collection of $(n-1)$ hyperplanes, each perpendicular to a coordinate axis and each containing one of the integral lattice points. Consider the $2^{n}$ translates of this collection of planes, subject to the condition that each translate contains $n$ hyperplanes whose intersection is a corner of the $n$-cube $[0,1 / 2]^{n} \subset E^{n}$; let these translates be ordered in some fashion and let $A(i), i=1,2, \cdots, 2^{n}$, be the collection of complementary domains into which $E^{n}$ is divided by the underlying pointset of the $i$ th translate. Each $A(i)$ is a pair-wise disjoint collection of open $n$-cubes, with sides parallel to the coordinate axes and of unit length; $A=\bigcup A(i), i=1,2, \cdots$, is an open cover of $E^{n}$. If $a \in A(i)$, call $i$ the degree of $a, \operatorname{deg} a$.

THEOREM 1. The elements of the above open cover $A$ can be ordered to satisfy the a.c.c.; in fact, so that each ascending chain is also ascending in degree, and hence has length at most $2^{n}$.

Received by the editors January 24, 1969. 
Proof. Note that it is sufficient to insure that the ordering is locally ascending in degree at each $a \in A:$ if $b \in A, b \cap a \neq \varnothing$, and $\operatorname{deg} b<\operatorname{deg} a$, then $b$ precedes $a$ in the ordering.

Select some element of $A\left(2^{n}\right)$ as base element. Begin the ordering of $A$ by listing all elements of $A(1)$ which can be chained to the base element by chains [not necessarily ascending] of elements of $A$ of length at most $2^{n}$. Next list all elements of $A(2)$ which can be connected to the base element by chains of elements of $A$ of length at most $2^{n}-1$. Proceed, at the $i$ th stage listing the elements of $A(i)$ which can be connected to the base element by chains of length at most $2^{n}-(i-1)$; end the listing corresponding to this choice of base element by listing the base element itself, as the sole element of $A\left(2^{n}\right)$ listed. Now another element of $A\left(2^{n}\right)$ is selected as base element and the same listing procedure applied, except that elements already listed are not listed again. Then a third base element is selected from $\mathrm{A}\left(2^{n}\right)$ and the procedure repeated, etc. The base elements are to be chosen systematically to exhaust $A\left(2^{n}\right)$, insuring that all of $A$ is ordered. Clearly, the ordering obtained is locally ascending in degree, completing the proof of the theorem.

Corollary 1. Let $n$ be a positive integer $\epsilon>0$, and $\delta=\epsilon / 2^{n+1} \sqrt{ } n$. If $U$ is a locally finite collection of subsets of $E^{n}$, each of diameter less than $\delta$, then the elements of $U$ may be ordered to satisfy the a.c.c.; in fact the ordering may be chosen so that each ascending chain has diameter at most $\epsilon$.

Proof. As in [4, Lemma 1.1], consider a cover $A^{\prime}$ of $E^{n}$, identical with $A$ above, except that the cubes have side $2 \epsilon$ rather than unity. $\delta$ is a Lebesgue number for this cover, and $\epsilon$ is an upper bound for the diameters of chains of length $2^{n}$. Order $A^{\prime}$ by Theorem 1 , and order $U$ by first listing the elements of $U$ which lie in the first element of $A^{\prime}$, next those not yet listed which lie in the second element, etc. Since an ascending chain of $U$ lies in an ascending chain of $A^{\prime}$, its diameter is at most $\epsilon$.

A null sequence of sets in a metric space is a countable collection such that, for each $\eta>0$, only finitely many sets of the collection have diameter greater than $\eta$.

Theorem 2. Suppose $X$ is a finite dimensional, locally compact, separable metric space, $V$ is an open subset of $X$, and $U$ is a collection of compact subsets of $V$ which is locally finite with respect to points of $V$ and which forms a null sequence in the one-point compactification of $X$. Then $U$ may be ordered so that the a.c.c. is satisfied and the collection of 
ascending chains is itself a null sequence in the one-point compactification.

Proof. Assume that $X$ is compact; otherwise, replace it by its one-point compactification. Since $X$ is finite dimensional, suppose $X$ is a subset of some $E^{n}$; in fact, that $V \subset$ int $B(1)$ and $X-V \subset S(1)$, where $B(r)$ is the $n$-ball centered at the origin of radius $r$, and $S(r)$ is its boundary. A radial, nonuniformly-expansive homeomorphism $h$ : int $B(1) \rightarrow E^{n}$ will be defined, such that diam $h(u) \leqq 3, u \in U$; Corollary 1 then orders $\{h(u), u \in U\}$, hence $U$ also.

Let $\pi: B(1)-\{$ origin $\} \rightarrow S(1)$ be the radial projection and let $r_{1}$, $r_{2}, \ldots$ be a sequence of positive numbers increasing to 1 and satisfying the following:

(i) $r_{1}>1 / 2$ and if $u \in U$ hits the origin, then $u \subset B\left(r_{1}\right)$.

(ii) If $u \in U$ and $u \cap B\left(r_{i}\right) \neq \varnothing$, then $u \subset B\left(r_{i+1}\right)$.

(iii) If $\operatorname{diam} \pi(u) \geqq 1 / i$, then $u \subset B\left(r_{i}\right), u \in U$.

The desired homeomorphism $h$ takes $S\left(r_{i}\right)$ radially onto $S(i)$ and extends linearly on the radial segments between $S$ 's. If $u \in U$, then diam $h(u)$, measured radially, is at most 2 , and measured angularly is at most 1 . Hence diam $h(u) \leqq 3$. Now order $\{h(u), u \in U\}$, and thus $U$, by Corollary 1 ; the properties of $h$ assure that the collection of ascending chains is a null sequence.

Corollary 2. If $h_{i}: X \rightarrow X, i=1,2, \cdots$, is a homeomorphism which moves only points in $u_{i}$ (where $u_{1}, u_{2}, \cdots$ is the ordering of $U$ given by Theorem 2), then the sequence $h_{1}, h_{2} h_{1}, h_{3} h_{2} h_{1}, \ldots$ of homeomorphisms converges to a homeomorphism.

Proof. A compact set in $V$ hits only finitely many ascending chains in $U$; hence the limit of the compositions is a finite composition on each compact subset of $V$. This shows that the limit is well defined and one-to-one, and that it is continuous at points of $V$. Continuity at boundary points of $V$ follows readily from the fact that the collection of ascending chains is a null sequence.

While the partition of $E^{n}$ used in Theorem 1 resembles that frequently used in complexes, the condition of Theorem 2 is topological, independent of any triangulation or metric structure. Other known convergence criteria, as Anderson and Bing [1, Theorem 4.2], Bing [2, Theorem 7], McAuley [5, Theorem H], and Gehman [3, Theorem $1]$, generally require closer relations between their sequences of homeomorphisms and sequences of open sets, of covers, etc.

Question. Is finite dimensionality necessary in Theorem 2? Might not some infinite dimensional spaces satisfy it, while others fail to? 
A space which is the disjoint union of finite dimensional spaces seems a candidate to satisfy; the Hilbert cube probably does not.

\section{REFERENCES}

1. R. D. Anderson and R. H. Bing, A complete elementary proof that Hilbert space is homeomorphic to the countable infinite product of lines, Bull. Amer. Math. Soc. 74 (1968), 771-792.

2. R. H. Bing, Each disk in $E^{3}$ contains a tame arc, Amer. J. Math. 84 (1962), 583590.

3. H. M. Gehman, Concerning sequences of homeomorphisms, Proc. Nat. Acad. Sci. U. S. A. 18 (1932), 460-465.

4. D. S. Gillman, Unknotting 2-manifolds in 3-hyperplanes of $E^{4}$, Duke Math. I. 33 (1966), 229-245.

5. L. F. McAuley, Some upper semi-continuous decompositions of $E^{3}$ into $E^{3}$. Ann. of Math. (2) 73 (1961), 437-457.

Rutgers, The State University 